\title{
Research Article \\ Study of UV Transmission through a Few Edible Oils and Chicken Oil
}

\author{
K. Anil Kumar and K. Viswanathan \\ Department of Physics, Karpagam University, Eachanari Post, Coimbatore 641021, Tamil Nadu, India \\ Correspondence should be addressed to K. Viswanathan; kvnooty@gmail.com
}

Received 17 June 2012; Revised 21 October 2012; Accepted 5 November 2012

Academic Editor: Jose Sousa Camara

Copyright (C) 2013 K. Anil Kumar and K. Viswanathan. This is an open access article distributed under the Creative Commons Attribution License, which permits unrestricted use, distribution, and reproduction in any medium, provided the original work is properly cited.

\begin{abstract}
Effects of solar UV radiation on the biosphere are well known and a lot of studies are going on to reduce its dangerous effects on human beings. Atmospheric ozone layer is not uniform over the globe, so that less ozone over tropics makes it receive more UV insolation, than high latitude places. People in the tropics are continuously exposed to high UV dosage, leading to skin reddening and the dangerous "sun stroke." In southern India people use some vegetable oils to protect the skin from sun heat. Studies of "sun stroke" show that people having skin of moderate colour are the main victims. In some parts of Southern Kerala like Kottayam, Ernakulam, and Alappuzha traditional people were using oil extracted from chicken, called "chicken oil," for the treatment of "heat burns" and other types of burns. This motivated us to study the UV absorption characteristics of chicken oil, and compare them with that of other commonly used vegetable oils. It is found that the chicken oil shows maximum absorption of UV radiation of about $85 \%$, and neem oil shows absorption of about $60 \%$. Other oils show comparatively very low absorption of UV radiation. So this property of chicken oil may be the reason for using it as remedy for heat burns.
\end{abstract}

\section{Introduction}

Effects of solar ultraviolet (UV) radiation on the biosphere are well known. In an era of atmospheric ozone depletion, there is concern about the solar UV radiation affecting human health. Ozone present in the earth atmosphere absorbs completely the solar radiation of wavelengths below $290 \mathrm{~nm}$ and protects our biosphere from this harmful radiation. Any reduction in atmospheric ozone causes an increase in the UV radiations, and hence the harms due to that increases the harms due to the radiations [1-3]. The existence of lower total columnar ozone over the tropics lets this region receive relatively large dose of solar UV radiation throughout the year in relation to the regions in the higher latitudes. Over the tropics the total ozone column is nearly $235 \mathrm{DU}(1 \mathrm{DU}=1 \mathrm{mill} \cdot \mathrm{atm} \cdot \mathrm{cm})$ and increases highly at polar latitudes to a value of 450 DU. The ozone present in the northern Hemisphere, where most part of the land is existing, is $285 \mathrm{DU}$. At the same time, in the southern hemisphere, which is mostly water locked, the ozone present is about $300 \mathrm{DU}[4,5]$. Naturally, the population in the tropics is thus continuously exposed to a larger solar UV dosage. In the UVB region (280 to $320 \mathrm{~nm}$ ), the biological efficacy is maximum at $280 \mathrm{~nm}$, which gradually reduces to a minimum value at $320 \mathrm{~nm}$; the magnitude of this efficacy spans two decades [6]. Therefore, an increase in flux at lower wavelength will have a farreaching influence on all life forms. A decrease in total ozone may induce a shift in the cutoff wavelength towards the lower wavelength radiation reaching the surface. Thus, lower total ozone over the tropics not only increases the UV dosage, but also exposes the biosphere to a more harmful shorter wavelength radiation. Solar UVB radiation causes reddening of the skin called "erythema" on the Caucasian skin and therefore the radiation is called erythemal radiation [7]. The effect of solar UVB on the non-Caucasian skin is considered negligible due to the presence of the pigment called melanin. As the amount of solar UVB is high at the tropics where the non-Caucasian population is also present, it is worthwhile 
to study whether any other protection mechanism aids the population in the tropics, apart from the pigment.

1.1. Motivation. In the life style of the general population in the tropics, particularly in South India, certain vegetable oils are applied on the head and body, well before taking bath. There might have been different reasons for the use of such oils as ointment on the body and skin. We examined the UV transmission properties of a few of these oils in common use among the people in the tropical India to find out whether these oils could have played a role in reducing the UVB exposure. In some area of Southern Kerala like Kottayam, Ernakulam, and Alappuzha, traditional families use oil extracted from chicken called "chicken oil" for the treatment of "heat burns" and other burns including "sun burns." This motivated the authors to study the UV absorption effects of the chicken oil, and to compare with that of other vegetable oils and cod liver oil. From this study alone, we cannot quantify their actual role in the protection of the skin of the population living in the tropics from the UVB radiation. More experiments, involving the population, need to be conducted to confirm such inference. This study is restricted to the examination of UV transmission characteristics of oils.

\section{Experimental}

2.1. Sample Preparation. The samples used for the study are mainly oils, regularly used in southern parts of India, especially in Tamil Nadu and Kerala. The oils used for the study are coconut oil, neem oil, sesame oil, castor oil, groundnut oil, cod liver oil, mustard oil and chicken oil. The coconut oil was prepared from coconut milk which was extracted from matured coconuts. The other vegetable oils were bought from the local markets as branded products. So the purity of the oils may be considered as approximately $90 \%$. Cod liver oil was also bought as a branded product. But the chicken oil was prepared from chicken carcass after removing the meat. The parts of chicken were boiled up to 140 degree Celsius and the oil was extracted from it. The oil has a pale yellow colour, with a chicken smell [5]. All the sample oils were collected in clean and dried bottles, so there is no contamination of samples. Therefore the results obtained can be considered as accurate.

2.2. Method of Measurement. A dual beam UV-Visible spectrophotometer was used to study the transmission characteristics of these oils. Commercially available samples of the oils were used in this study. The study was conducted at CUSAT, Cochin, and also at STIC, Cochin.

As a preliminary step all samples of oils were taken in a UV transparent quartz cell. The dimension of the cell is $5 \times$ $5 \times 10 \mathrm{~mm}$, which provides a path length of $5 \mathrm{~mm}$ for UV-B radiations. The transmission characteristics of these oils were taken using a dual beam UV spectrophotometer.

The measurements were taken one by one after cleaning the quartz cell before taking each sample.
To start with, the transmission of the blank quartz disk was taken. Later, one of the oils was smeared and the transmission of the disk with the oil was taken. Then the disk was cleaned and the transmission of the disk alone was taken to ensure that no trace of the oil is left on the disk. Then the second oil was smeared and the procedure repeated.

The experiment was repeated with a quartz disc of $2 \mathrm{~mm}$ thick and approximately $25 \mathrm{~mm}$ diameter. A thin layer of oil was smeared on the disc and transmission recorded. The thickness of the oil may be considered as being approximately similar to the thickness of oil applied over the skin. The characteristics of the Quartz disk were taken before smearing each and every oil sample and compared with characteristics taken with the blank disk. This is to ensure that there is no contamination of the samples. Oil smears had thickness well less than $1 \mathrm{~mm}$.

\section{Results and Discussion}

The absorption of UV visible corresponds to the excitation of outer electrons. There are three types of electronic transitions which can be considered:

(1) transitions involving $\pi, \sigma$, and $n$ electrons,

(2) transitions involving charge-transfer electrons,

(3) transitions involving $d$ and $f$ electrons.

Absorption of UV radiation in organic molecules is restricted to certain functional groups (chromophores) that contain valence electrons of low excitation energy. The spectrum of a molecule containing these chromophores is complex. This is because the superposition of rotational and vibrational transitions on the electronic transitions gives a combination of overlapping lines. This appears as a continuous absorption band.

$\sigma \rightarrow \sigma^{*}$ Transitions. An electron in a bonding $\sigma$ orbital is excited to the corresponding antibonding orbital. The energy required is large. Absorption maxima due to $\sigma \rightarrow \sigma^{*}$ transition are not seen in UV-Vis spectra.

$n \rightarrow \sigma^{*}$ Transitions. Saturated compounds containing atoms with lone pairs (nonbonding electrons) are capable of $n \rightarrow$ $\sigma^{*}$ transitions. The energy is less than the above transitions. This can be initiated by light of wavelength 150-250 nm.

$n \rightarrow \pi^{*}$ and $\pi \rightarrow \pi^{*}$ Transitions. Most absorption spectroscopy of organic compounds is based on $n$ or $\pi$ electrons to the $\pi^{*}$ excited state. The absorption spectra for these transitions fall in the region of wavelength 200-700 nm. These transitions need an unsaturated group in the molecule to provide the $\pi$ electrons.

The study of UV absorption and transmission through oils was limited to the UVB region $(280-320 \mathrm{~nm}$ ) because of its dangerous effect on our skin. Of the eight oils used, 4 show poor absorption in the UVB region, while the other 3 oils show moderate absorption, but chicken oil shows very high absorption (Figure 1). Coconut, groundnut, cod liver oil, and mustard oil show transmission above nearly $100 \%$ in the UVB. Sesame oil shows $70 \%$ transmission. Castor oil 


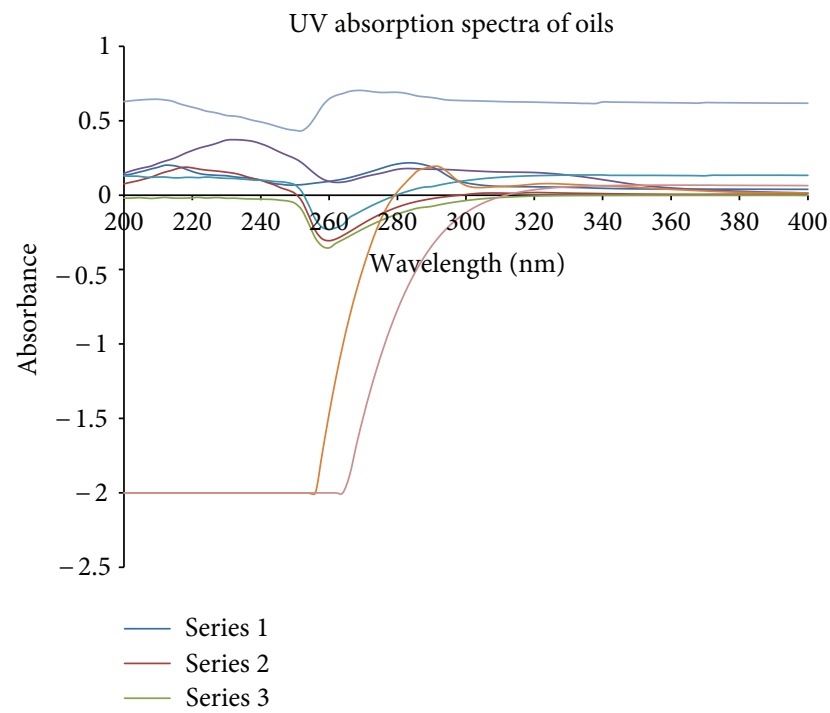

FIGURE 1: UV absorption spectra of vegetable oils, cod liver oil, and chicken oil.

shows $75 \%$ and neem oil shows $65 \%$ transmission. Chicken oil prepared from chicken wastage showed an exciting low transmission level of $25 \%$. This wonderful result showed by chicken oil is due to the large number of unsaturated chromophores present in it.

The UV absorption graph (Figure 1) shows wonderful results in UVB region. The cod liver oil and coconut oil show very low poor rate of absorption from -2 to 0 . Groundnut oil shows poor absorption of 0 . Mustard oil and castor oil show slight absorption of 0 to 0.2 . Sesame oil shows absorption of 0 to 0.25 . Neem oil which is used as medicine for skin shows some better absorption of 0.1 to 0.4 . But the wonderful result was shown by chicken oil. It shows a very high range of absorption of 0.6 to 0.75 .

From Figure 2 it can be seen that all eight oils show their transmission in UV rays of wavelengths 200 and $400 \mathrm{~nm}$. Coconut oil shows nearly $80 \%$ transmissions in UVB region. Cod liver oil shows 60 to $75 \%$ transmission. Groundnut oil and mustard oil show nearly $100 \%$ transmissions. Castor oil shows $75 \%$ and sesame oil shows nearly $80 \%$ transmission. Neem oil shows 60 to $70 \%$ transmission of UV rays. It may be due to the presence of more chromophores in these oils than others. Even though the components are equal in all oils, the property of lower transmission of UV rays, neem oil is used as a protection to human and animal skin in summer seasons.

Figures 1 and 2 show the UV absorption and transmission through oils. The wonderful characteristic in UV transmission and absorption of UV rays from $200 \mathrm{~nm}$ to $400 \mathrm{~nm}$ was produced by chicken oil. The average transmission of UV rays is nearly 20 to $25 \%$. This wonderful property of UV transmission is very useful for protecting human skin from sun stroke, which is due to the high dosage of UV rays from the sun. So, from the study of UV transmissions through different oils, it is clear that chicken oil is a very effective and

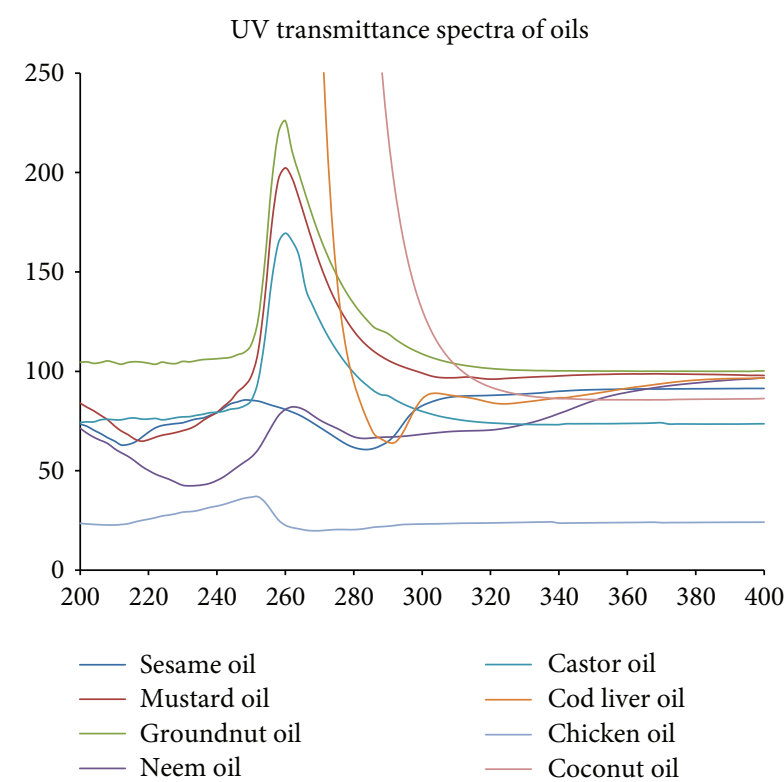

FIGURE 2: UV transmittance spectra of vegetable oils, cod liver oil, and chicken oil.

safe oil to protect skin from solar UV rays. It is also a very good skin so softener.

Table 1 shows characteristics of different oils, which we have studied and reported in this paper. It can be seen that coconut oil contains nearly $85 \%$ saturated oils like palmitic acid, stearic acid, and myristic acid. In sesame oil, castor oil, ground nut oil, and cod liver oil components are approximately the same, that is, saturated oils (18\%), monounsaturated oil (50\%), and polyunsaturated oil (32\%). But in mustard oil the composition is saturated oil (12\%), monounsaturated oil (60\%), and polyunsaturated oil (21\%); neem oil contains saturated oil (14\%), monounsaturated oil (53\%), and polyunsaturated oil (32\%).

Saturated fatty acids have no double bond between the individual carbon atoms of the fatty acid chain. That is, the chain of carbon atoms is fully "saturated" with hydrogen atoms. There are many kinds of naturally occurring saturated fatty acids, which differ mainly in number of carbon atoms, from 3 carbons (propionic acid) to 36 (hexatriacontanoic acid). Monounsaturated fatty acids have one double bond in the fatty acid chain and all of the remaining carbon atoms in the chain are single bonded. By contrast, polyunsaturated fatty acids have more than one double bond. Polyunsaturated fatty acids possess both cis and trans isomerism depending on the geometry of the molecule. Major monounsaturated fatty acid is oleic acid and major polyunsaturated fatty acid is linoleic acid.

In saturated oils, all carbon atoms are connected with single strong $\sigma$-bonds. $\mathrm{C}-\mathrm{C}$ bonds are much stronger and electrons need very high energy to break the bond for excitation. So oils consisting of saturated fatty acids as the major part are not able to absorb energy in shorter wavelengths. Absorption maxima of $\sigma-\sigma^{*}$ transition need large energy and it is not possible in UV-Vis spectra. So coconut oil does not 
TABLE 1: Characteristics of different oils.

\begin{tabular}{|c|c|c|c|c|c|}
\hline Name of oil & Density $\left(\mathrm{kg} / \mathrm{m}^{3}\right)$ & Components & Colour & Odour & $\mathrm{pH}$ value \\
\hline & & Saturated oils (86\%) & & & \\
\hline \multirow[t]{3}{*}{ Coconut oil } & 924 & Monounsaturated oil (6\%) & Colourless & Smell of coconut & 4 to 6 \\
\hline & & Polyunsaturated oil (4\%) & & & \\
\hline & & Saturated oils (18\%) & & & \\
\hline \multirow[t]{3}{*}{ Sesame oil } & 923 & Monounsaturated oil (45\%) & Yellow & Odourless & 7 to 7.5 \\
\hline & & Polyunsaturated oil (32\%) & & & \\
\hline & & Saturated oils (17\%) & & & \\
\hline \multirow[t]{3}{*}{ Ground nut oil } & 913 & Monounsaturated oil (46\%) & Pale yellow & Odourless & 6 to 7 \\
\hline & & Polyunsaturated oil (32\%) & & & \\
\hline & & Saturated oils (12\%) & & & \\
\hline \multirow[t]{3}{*}{ Mustard oil } & 936 & Monounsaturated oil (60\%) & Pale brown & Unpleasant smell & 5 to 6.5 \\
\hline & & Polyunsaturated oil (21\%) & & & \\
\hline & & Saturated oils (15\%) & & & \\
\hline \multirow[t]{3}{*}{ Castor oil } & 961 & Monounsaturated oil (47\%) & Pale yellow & Unpleasant smell & 4 to 5 \\
\hline & & Polyunsaturated oil (22\%) & & & \\
\hline & & Saturated oils (14\%) & & & \\
\hline \multirow[t]{3}{*}{ Neem oil } & 935 & Monounsaturated oil (53\%) & Brown & Garlic & 6.5 to 7.5 \\
\hline & & Polyunsaturated oil (32\%) & & & \\
\hline & & Saturated oils (18\%) & & & \\
\hline \multirow[t]{3}{*}{ Cod liver oil } & 929 & Monounsaturated oil (50\%) & Pale yellow & Smell of fish & 5.5 to 6.5 \\
\hline & & Polyunsaturated oil (32\%) & & & \\
\hline & & Saturated oils (7\%) & & & \\
\hline \multirow[t]{2}{*}{ Chicken oil } & 934 & Monounsaturated oil (58\%) & Yellow & Smell of chicken & 6 to 7 \\
\hline & & Polyunsaturated oil (34\%) & & & \\
\hline
\end{tabular}

absorb UV rays and transmits most of the rays. But due to the presence of small number of nonbonding electrons $n$ $\sigma^{*}$ transition is possible, so that coconut oil shows some absorptions in higher wavelength of UV radiations.

Other oils like sesame oil, ground nut oil, and cod liver oil contain $70 \%$ unsaturated fatty acids-both monounsaturated (one $\mathrm{C}-\mathrm{C}$ double bond) and polyunsaturated (two or more $\mathrm{C}-\mathrm{C}$ double bonds). So $n-\pi^{*}$ and $\pi-\pi^{*}$ transitions are possible due to the presence of $\pi$-bonds. These oils show more absorption of UV rays than coconut oil. Mustard oil and neem oil show more absorption because these oils contain nearly $82 \%$ of unsaturated fatty acids, and have more number of $\pi$ electrons than other oils. So more number of $\pi-\pi *$ electronic transfer is possible in these oils, and hence they absorb more UV rays than others. Chicken oil contains nearly $90 \%$ unsaturated fatty acids (both monounsaturated and polyunsaturated) and a large number of unsaturated molecules are present. Beer's law states that

$$
A=\varepsilon c l,
$$

where $A$ is the absorption, $\varepsilon$ is the molar absorptivity (extinction coefficient), $c$ is the concentration of sample, and $l$ is the path length of the radiation in the sample; if the absorption is stronger, the value of $\varepsilon$ will also be higher.

Conjugated molecules absorb energy easily than the isolated double bonds because the energy gap of $\pi-\pi^{*}$ for the electronic transfer is very small. In chicken oil a large number of conjugated molecules are present and they absorb energy even from lower wavelengths of UV-Vis spectrum. Only conjugated alkenes molecules cause shift in $\lambda_{\max }$ (maximum wavelength); as the conjugation increases, the shift also increases. So, the presence of large number of molecules of conjugated unsaturated alkenes in chicken oil is responsible for the absorption of large UV radiations by it.

\section{Conclusions}

The UV-Vis absorption studies of chicken oil, mustard oil, sesame oil, neem oil, coconut oil, cod liver oil, castor oil, and ground nut oil show that most of the vegetable oils show poor absorption of UV radiations, but chicken oil, neem oil, and castor oil show considerably high absorption rate. The wonderful absorption, nearly $75 \%$, of chicken oil is highly interesting. This suggests that the oil may be used for prevention of UV radiation problems on human skin.

\section{Acknowledgments}

The authors are thankful to the staff of STIC (Sophisticated Test and Instrumentation Centre) CUSAT, for helping to record the UV spectra. Also, they extend their thanks to the 
Management of Karpagam University, for giving them the facilities.

\section{References}

[1] L. Aldez, "Flux measurements of atmospheric ozone over land and water," Journal of Geophysical Research, vol. 74, no. 28, pp. 6943-6946, 1969.

[2] T. J. Barton and G. W. Pattridge, "The Australian Climatology of Biologically Effective Ultraviolet Radiation," The Australian journal of Dermatology, vol. 20, no. 2, pp. 89-74, 1979.

[3] K. Asif Biswas, The Ozone Layer, Pergamon Press, Washington, DC, USA, 1979.

[4] E. Meszaros, Atmospheric Chemistry: Fundamental Aspects, Elsevier, New York, NY, USA, 1981.

[5] Ozone Data for the World, World Ozone Data Centre, Toronto, Canada, 2001.

[6] H. Walter, Biological Effects of Ultraviolet Radiation, Cambridge University Press, Cambridge, UK, 1980.

[7] S. Teressa, "UV transmission through a few edible oils in the context of changing solar insolation," The Journal of Indian Geophysical Union, vol. 8, no. 4, pp. 261-271, 2004. 

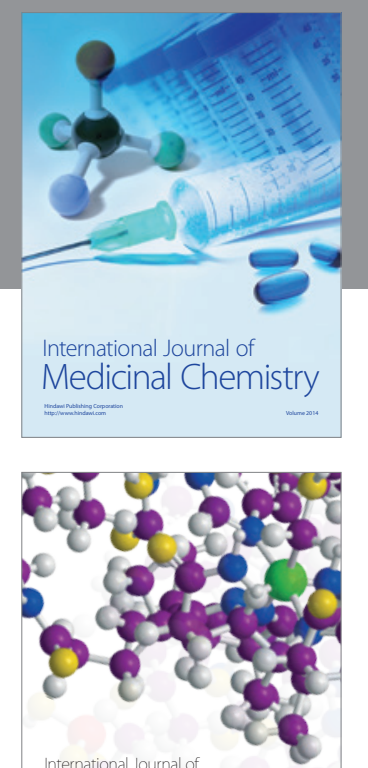

\section{Carbohydrate} Chemistry

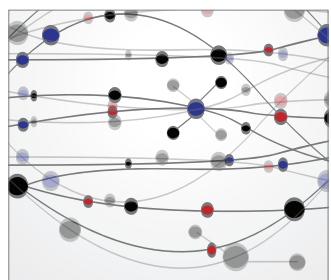

The Scientific World Journal
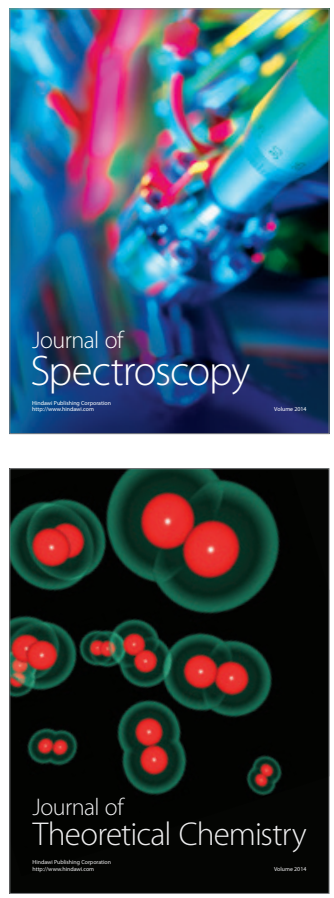
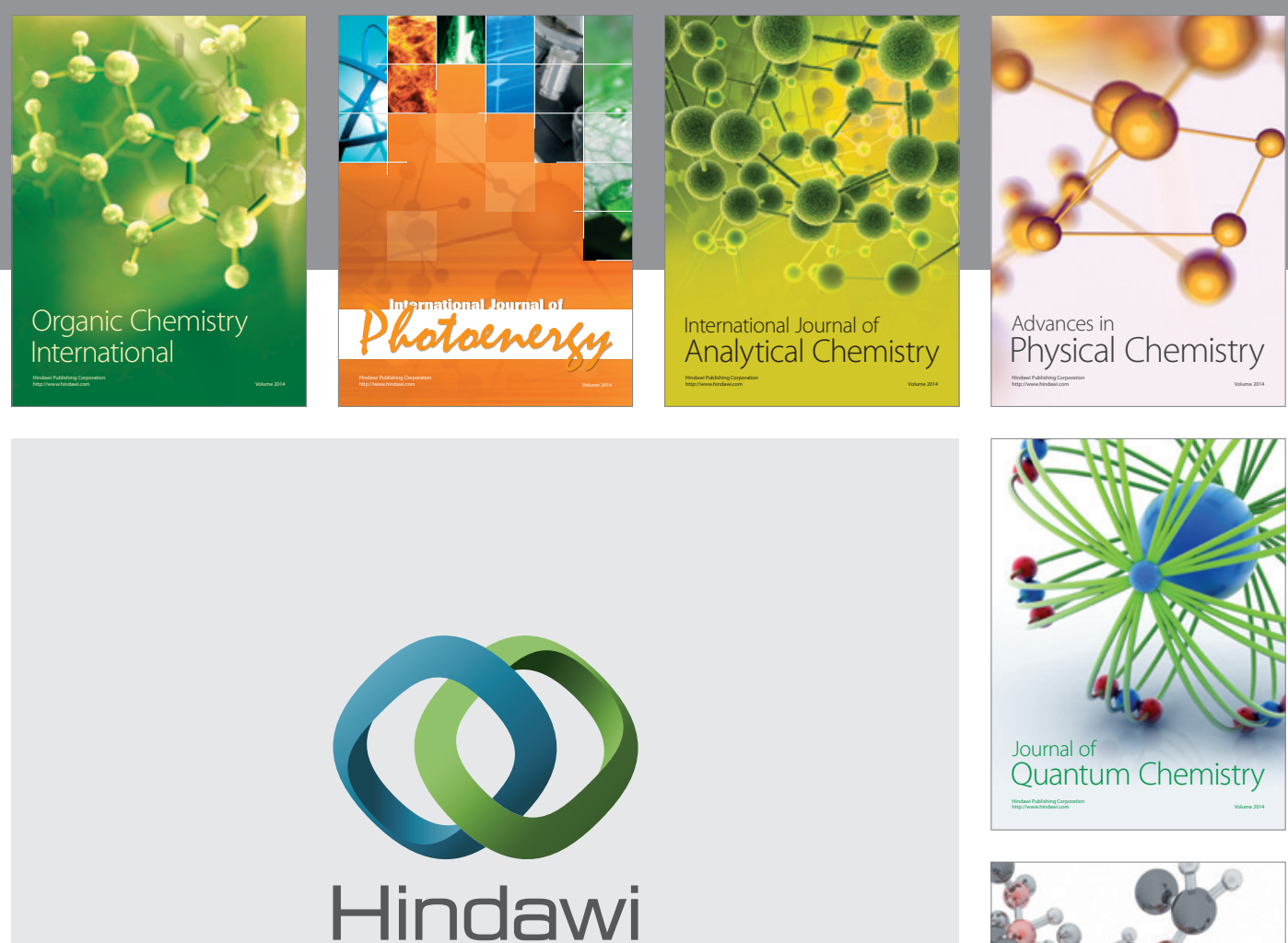

Submit your manuscripts at

http://www.hindawi.com

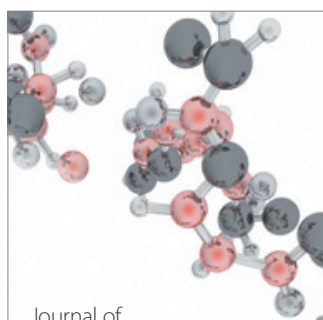

Analytical Methods

in Chemistry

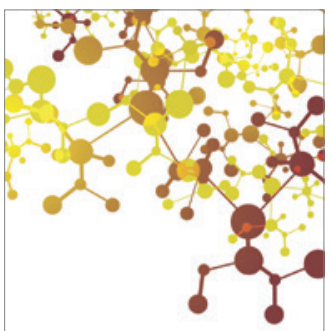

Journal of

Applied Chemistry

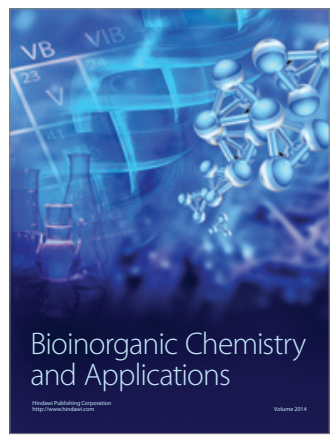

Inorganic Chemistry
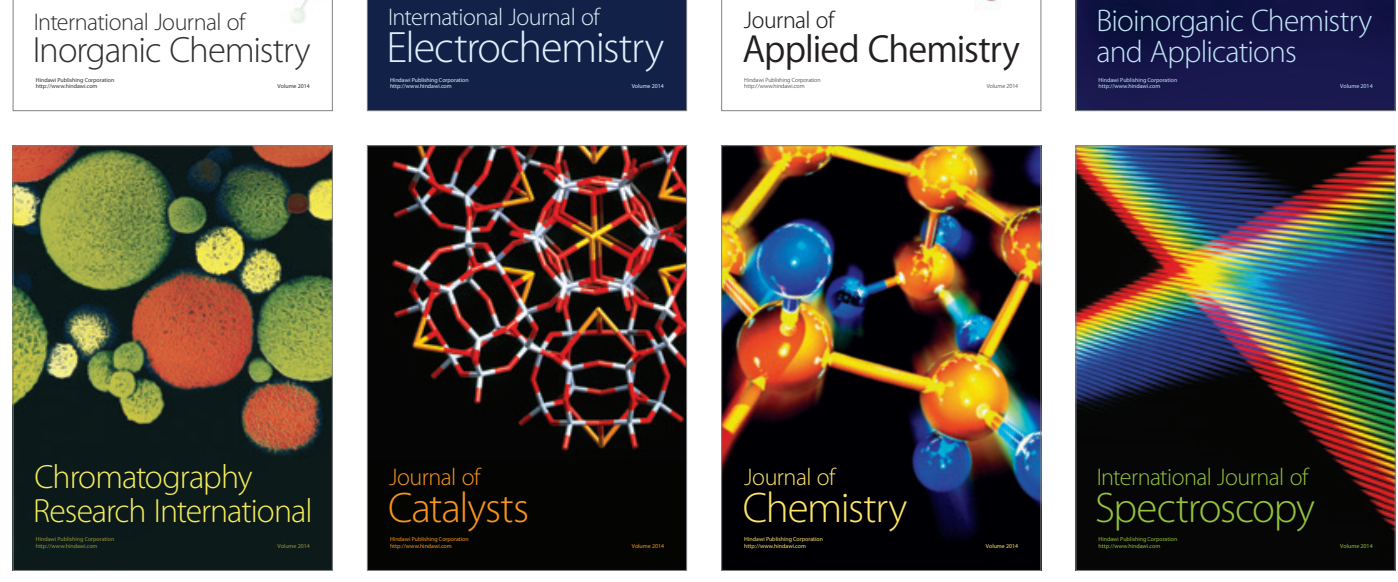